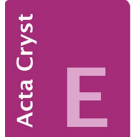
COMMUNICATIONS

ISSN 2056-9890

\section{Crystal structure of the borabenzene- 2,6-lutidine adduct}

\author{
Lauri Kivijärvi and Matti Haukka*
}

Department of Chemistry, University of Jyväskylä, PO Box 35, Fl-40014 Jyväskylä, Finland. *Correspondence e-mail: matti.o.haukka@jyu.fi

Received 18 October 2015; accepted 30 October 2015

Edited by W. T. A. Harrison, University of Aberdeen, Scotland

In the title compound, $\mathrm{C}_{12} \mathrm{H}_{14} \mathrm{BN}$, the complete molecule is generated by a crystallographic twofold axis, with two $\mathrm{C}$ atoms, the $\mathrm{B}$ atom and the $\mathrm{N}$ atom lying on the rotation axis. The dihedral angle between the borabenzene and pyridine rings is $81.20(6)^{\circ}$. As well as dative electron donation from the $\mathrm{N}$ atom to the $\mathrm{B}$ atom $[\mathrm{B}-\mathrm{N}=1.5659$ (18) $\AA$ ] , the methyl substituents on the lutidine ring shield the $\mathrm{B}$ atom, which further stabilizes the molecule. In the crystal, weak aromatic $\pi-\pi$ stacking between the pyridine rings [centroid-centroid separation $=3.6268(9) \AA]$ is observed, which generates [001] columns of molecules.

Keywords: crystal structure; borabenzene; $\pi-\pi$ stacking.

CCDC reference: 1434350

\section{Related literature}

For the synthesis of the title compound, see: Hoic et al. (1996). For a related structure, see: Boese et al. (1985). For borabenzene adducts as analogues of cyclopentadienyl anions (Cp), see: Bazan et al. (2000); Wang et al. (2002); Cui et al. (2010). For the uses of borabenzenes and their metal complexes, see: Wang et al. (2002; Jaska et al. (2006).

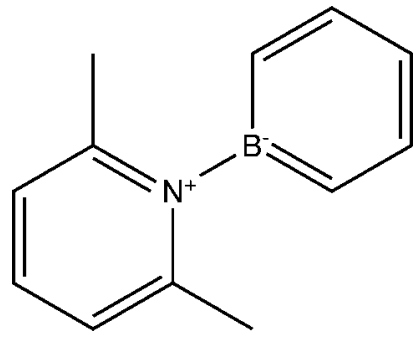

\section{Experimental}

2.1. Crystal data

$\mathrm{C}_{12} \mathrm{H}_{14} \mathrm{BN}$

$M_{r}=183.05$

Monoclinic, $C 2 / c$

$a=10.008(2) \AA$

$b=14.447(3) \AA$

$c=7.1360$ (14) $\AA$

$\beta=90.16(3)^{\circ}$

$$
\begin{aligned}
& V=1031.8(4) \AA^{3} \\
& Z=4 \\
& \text { Mo } K \alpha \text { radiation } \\
& \mu=0.07 \mathrm{~mm}^{-1} \\
& T=120 \mathrm{~K} \\
& 0.24 \times 0.18 \times 0.16 \mathrm{~mm}
\end{aligned}
$$

\subsection{Data collection}

Bruker Kappa APEXII CCD diffractometer

Absorption correction: multi-scan (SADABS; Bruker, 2012)

$$
T_{\min }=0.646, T_{\max }=0.746
$$

\subsection{Refinement}

$R\left[F^{2}>2 \sigma\left(F^{2}\right)\right]=0.044$

$w R\left(F^{2}\right)=0.120$

$S=1.06$

1482 reflections

7258 measured reflections 1482 independent reflections 1280 reflections with $I>2 \sigma(I)$ $R_{\text {int }}=0.028$

Data collection: COLLECT (Bruker, 2008); cell refinement: DENZO/SCALEPACK (Otwinowski \& Minor, 1997); data reduction: DENZO/SCALEPACK; program(s) used to solve structure: SUPERFLIP (Palatinus \& Chapuis, 2007); program(s) used to refine structure: SHELXL2014 (Sheldrick, 2015); molecular graphics: CHIMERA (Pettersen et al., 2004); software used to prepare material for publication: SHELXL2014.

Supporting information for this paper is available from the IUCr electronic archives (Reference: HB7526).

\section{References}

Bazan, G. C., Cotter, W. D., Komon, Z. J. A., Lee, R. A. \& Lachicotte, R. J. (2000). J. Am. Chem. Soc. 122, 1371-1380.

Boese, R., Finke, N., Henkelmann, J., Maier, G., Paetzold, P., Reisenauer, H. P. \& Schmid, G. (1985). Chem. Ber. 118, 1644-1654.

Bruker (2008). COLLECT. Bruker AXS Inc., Madison, Wisconsin, USA

Bruker (2012). SADABS. Bruker AXS Inc., Madison, Wisconsin, USA.

Cui, P., Chen, Y., Zhang, Q., Li, G. \& Xia, W. (2010). J. Organomet. Chem. 695, 2713-2719.

Hoic, D. A., Wolf, J. R., Davis, W. M. \& Fu, G. C. (1996). Organometallics, 15 , 1315-1318.

Jaska, C. A., Emslie, D. J. H., Bosdet, M. J. D., Piers, W. E., Sorensen, T. S. \& Parvez, M. (2006). J. Am. Chem. Soc. 128, 10885-10896.

Otwinowski, Z. \& Minor, W. (1997). Methods in Enzymology, Vol. 276, Macromolecular Crystallography, Part A, edited by C. W. Carter Jr \& R. M. Sweet, pp. 307-326. New York: Academic Press.

Palatinus, L. \& Chapuis, G. (2007). J. Appl. Cryst. 40, 786-790.

Pettersen, E. F., Goddard, T. D., Huang, C. C., Couch, G. S., Greenblatt, D. M., Meng, E. C. \& Ferrin, T. E. (2004). J. Comput. Chem. 25, 1605-1612.

Sheldrick, G. M. (2008). Acta Cryst. A64, 112-122.

Sheldrick, G. M. (2015). Acta Cryst. C71, 3-8.

Wang, X., Zheng, X. \& Herberich, G. E. (2002). Eur. J. Inorg. Chem. 2002, 3141 


\section{supporting information}

Acta Cryst. (2015). E71, o944 [https://doi.org/10.1107/S2056989015020599]

\section{Crystal structure of the borabenzene-2,6-lutidine adduct}

\section{Lauri Kivijärvi and Matti Haukka}

\section{S1. Structural commentary}

The title compound lies on a two-fold rotational axis, which passes through atoms H3, C3, B1, N1, C6, and H6.

Borabenzene-2,6-lutidine is an example of nitrogen-stabilized borabenzene adducts. The nitrogen atom of the base (2,5lutidine) donates an electron pair to the boron atom, and thus stabilizes the borabenzene ring. Borabenzene-2,6-lutidine has a zwitterionic nature. The nitrogen ring bears a positive charge, and the boron ring a negative charge.

Borabenzenes are analogous to cyclopentadienyl anions ( $\mathrm{Cp}$ ), althought they are generally weaker electron donors than Cp (Bazan et al., 2000; Wang et al., 2002 and Cui et al., 2010). Borabenzene rings can thus be used as a replacement for $\mathrm{Cp}$ when weaker electron donation properties are required. There is a growing interest to utilize borabenzenes and their metal complexes in several applications including catalytic and semiconducting materials as well as light-emitting devices (Wang et al., 2002 and Jaska et al., 2006).

\section{S2. Synthesis and crystallization}

The compound was synthesized according to the previously reported procedure (Hoic et al. 1996). X-ray quality crystals were obtained by using the following procedure: In a glove box, borabenzene-2,6-lutidine was dissolved in pure toluene at room temperature until a saturated solution was obtained. The clear solution was separated and and the solution was allowed to evaporate slowly. Formed crystals were collected from the solution after one week and were immediately taken to an X-ray diffraction analysis. In order to protect the crystals from air and moisture, the crystals were immersed to cryo oil before taking them out from the glove box.

\section{S3. Refinement}

Crystal data, data collection and structure refinement details are summarized in Table 1. Hydrogen atoms were positioned geometrically and constrained to ride on their parent atoms, with $\mathrm{C}-\mathrm{H}=0.95-0.98 \AA$ and $\mathrm{U}_{\mathrm{iso}}=1.2-1.5 \mathrm{U}_{\mathrm{eq}}$ (parent atom). The highest peak is located $0.69 \AA$ from atom C5 and the deepest hole is located $0.67 \AA$ from atom N1. 


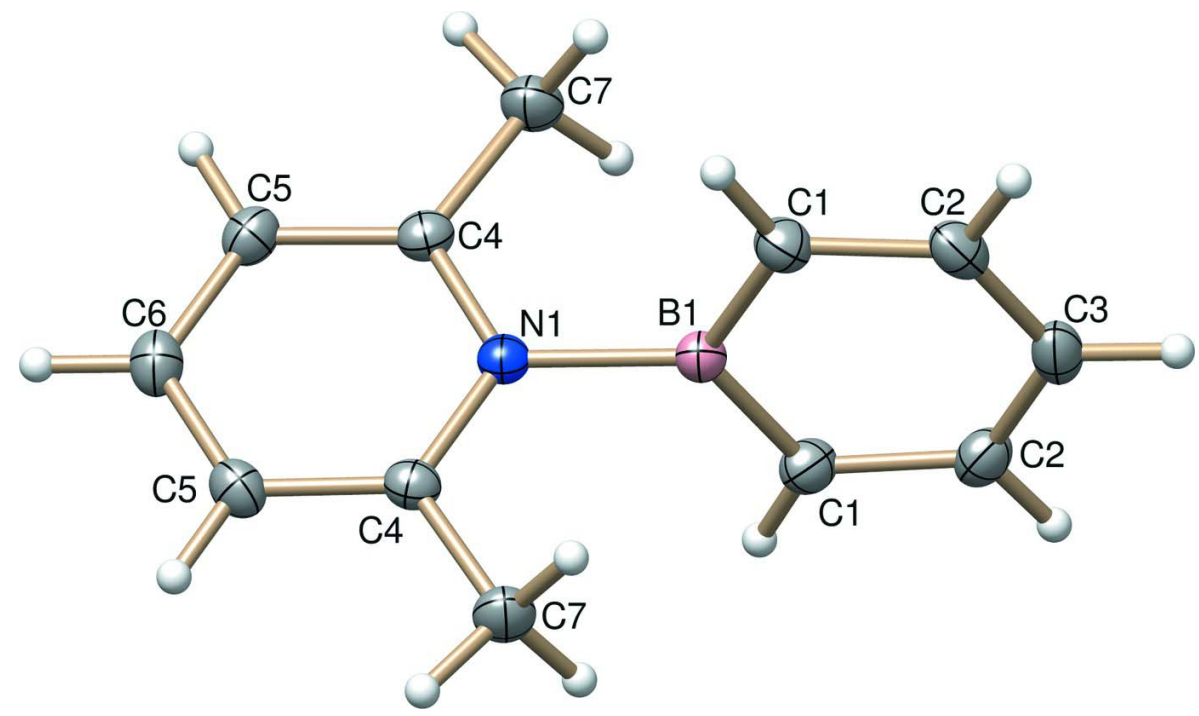

Figure 1

The molecular structure of the title compound, with atom labels and $50 \%$ probability displacement ellipsoids for non- $\mathrm{H}$ atoms.

Borabenzene-2,6-lutidine

Crystal data

$\mathrm{C}_{12} \mathrm{H}_{14} \mathrm{BN}$

$M_{r}=183.05$

Monoclinic, $C 2 / c$

$a=10.008(2) \AA$

$b=14.447$ (3) $\AA$

$c=7.1360$ (14) $\AA$

$\beta=90.16(3)^{\circ}$

$V=1031.8(4) \AA^{3}$

$Z=4$

\section{Data collection}

\section{Bruker Kappa APEXII CCD} diffractometer

Radiation source: fine-focus sealed tube

Horizontally mounted graphite crystal monochromator

Detector resolution: 16 pixels $\mathrm{mm}^{-1}$ $\varphi$ scans and $\omega$ scans with $\kappa$ offset Absorption correction: multi-scan (SADABS; Bruker, 2012)

Refinement

Refinement on $F^{2}$

Least-squares matrix: full

$R\left[F^{2}>2 \sigma\left(F^{2}\right)\right]=0.044$

$w R\left(F^{2}\right)=0.120$

$S=1.06$

1482 reflections
$F(000)=392$

$D_{\mathrm{x}}=1.178 \mathrm{Mg} \mathrm{m}^{-3}$

Mo $K \alpha$ radiation, $\lambda=0.71073 \AA$

Cell parameters from 10098 reflections

$\theta=1.0-28.7^{\circ}$

$\mu=0.07 \mathrm{~mm}^{-1}$

$T=120 \mathrm{~K}$

Needle, yellow

$0.24 \times 0.18 \times 0.16 \mathrm{~mm}$

$T_{\min }=0.646, T_{\max }=0.746$

7258 measured reflections

1482 independent reflections

1280 reflections with $I>2 \sigma(I)$

$R_{\text {int }}=0.028$

$\theta_{\max }=30.0^{\circ}, \theta_{\min }=4.0^{\circ}$

$h=-14 \rightarrow 14$

$k=-16 \rightarrow 20$

$l=-9 \rightarrow 9$

67 parameters

0 restraints

Hydrogen site location: inferred from neighbouring sites

$\mathrm{H}$-atom parameters constrained 
$w=1 /\left[\sigma^{2}\left(F_{\mathrm{o}}^{2}\right)+(0.0555 P)^{2}+0.6142 P\right]$

where $P=\left(F_{\mathrm{o}}^{2}+2 F_{\mathrm{c}}^{2}\right) / 3$

$(\Delta / \sigma)_{\max }<0.001$

$$
\Delta \rho_{\max }=0.30 \mathrm{e} \AA^{-3}
$$

\section{Special details}

Geometry. All e.s.d.'s (except the e.s.d. in the dihedral angle between two 1.s. planes) are estimated using the full covariance matrix. The cell e.s.d.'s are taken into account individually in the estimation of e.s.d.'s in distances, angles and torsion angles; correlations between e.s.d.'s in cell parameters are only used when they are defined by crystal symmetry. An approximate (isotropic) treatment of cell e.s.d.'s is used for estimating e.s.d.'s involving l.s. planes.

Fractional atomic coordinates and isotropic or equivalent isotropic displacement parameters $\left(\hat{A}^{2}\right)$

\begin{tabular}{lllll}
\hline & $x$ & $y$ & $z$ & $U_{\text {iso }} * U_{\text {eq }}$ \\
\hline N1 & 0.5000 & $0.38254(7)$ & 0.7500 & $0.0182(2)$ \\
C1 & $0.43941(9)$ & $0.22210(7)$ & $0.59114(13)$ & $0.0229(2)$ \\
H1 & 0.3993 & 0.2534 & 0.4883 & $0.028^{*}$ \\
C2 & $0.44362(10)$ & $0.12556(7)$ & $0.59879(14)$ & $0.0246(2)$ \\
H2 & 0.4069 & 0.0910 & 0.4977 & $0.030^{*}$ \\
C3 & 0.5000 & $0.07806(9)$ & 0.7500 & $0.0254(3)$ \\
H3 & 0.5000 & 0.0123 & 0.7500 & $0.031^{*}$ \\
C4 & $0.38816(9)$ & $0.42921(7)$ & $0.80474(12)$ & $0.0197(2)$ \\
C5 & $0.38693(9)$ & $0.52523(7)$ & $0.80370(13)$ & $0.0225(2)$ \\
H5 & 0.3086 & 0.5578 & 0.8398 & $0.027^{*}$ \\
C6 & 0.5000 & $0.57352(9)$ & 0.7500 & $0.0241(3)$ \\
H6 & 0.5000 & 0.6393 & 0.7500 & $0.029^{*}$ \\
C7 & $0.26941(10)$ & $0.37460(7)$ & $0.86747(15)$ & $0.0258(2)$ \\
H7A & 0.2957 & 0.3332 & 0.9699 & $0.039^{*}$ \\
H7B & 0.2351 & 0.3379 & 0.7624 & $0.039^{*}$ \\
H7C & 0.1996 & 0.4169 & 0.9112 & $0.039^{*}$ \\
B1 & 0.5000 & $0.27415(10)$ & 0.7500 & $0.0195(3)$ \\
& & & &
\end{tabular}

Atomic displacement parameters $\left(\AA^{2}\right)$

\begin{tabular}{lllllll}
\hline & $U^{11}$ & $U^{22}$ & $U^{33}$ & $U^{12}$ & $U^{13}$ & $U^{23}$ \\
\hline $\mathrm{N} 1$ & $0.0180(5)$ & $0.0187(5)$ & $0.0179(5)$ & 0.000 & $-0.0021(4)$ & 0.000 \\
$\mathrm{C} 1$ & $0.0222(4)$ & $0.0228(5)$ & $0.0239(5)$ & $-0.0022(3)$ & $-0.0018(3)$ & $0.0003(3)$ \\
$\mathrm{C} 2$ & $0.0222(4)$ & $0.0235(5)$ & $0.0282(5)$ & $-0.0047(3)$ & $0.0024(4)$ & $-0.0045(4)$ \\
$\mathrm{C} 3$ & $0.0230(6)$ & $0.0179(6)$ & $0.0355(7)$ & 0.000 & $0.0068(5)$ & 0.000 \\
$\mathrm{C} 4$ & $0.0183(4)$ & $0.0224(4)$ & $0.0183(4)$ & $0.0011(3)$ & $-0.0013(3)$ & $0.0003(3)$ \\
$\mathrm{C} 5$ & $0.0237(5)$ & $0.0221(5)$ & $0.0218(5)$ & $0.0042(3)$ & $0.0006(3)$ & $-0.0004(3)$ \\
$\mathrm{C} 6$ & $0.0309(7)$ & $0.0193(6)$ & $0.0220(6)$ & 0.000 & $-0.0004(5)$ & 0.000 \\
$\mathrm{C} 7$ & $0.0195(4)$ & $0.0268(5)$ & $0.0312(5)$ & $-0.0010(3)$ & $0.0025(4)$ & $-0.0003(4)$ \\
$\mathrm{B} 1$ & $0.0177(6)$ & $0.0177(6)$ & $0.0230(6)$ & 0.000 & $-0.0002(5)$ & 0.000 \\
\hline
\end{tabular}

Geometric parameters $\left(\AA,{ }^{\circ}\right)$

\begin{tabular}{llll}
\hline $\mathrm{N} 1-\mathrm{C} 4 \mathrm{i}$ & $1.3647(11)$ & $\mathrm{C} 4-\mathrm{C} 5$ & $1.3874(13)$ \\
$\mathrm{N} 1-\mathrm{C} 4$ & $1.3647(11)$ & $\mathrm{C} 4-\mathrm{C} 7$ & $1.4961(13)$ \\
$\mathrm{N} 1-\mathrm{B} 1$ & $1.5659(18)$ & $\mathrm{C} 5-\mathrm{C} 6$ & $1.3843(12)$
\end{tabular}




\begin{tabular}{|c|c|c|c|}
\hline $\mathrm{C} 1-\mathrm{C} 2$ & $1.3964(14)$ & $\mathrm{C} 5-\mathrm{H} 5$ & 0.9500 \\
\hline $\mathrm{C} 1-\mathrm{B} 1$ & $1.4881(12)$ & $\mathrm{C} 6-\mathrm{C}^{\mathrm{i}}$ & $1.3843(12)$ \\
\hline $\mathrm{C} 1-\mathrm{H} 1$ & 0.9500 & $\mathrm{C} 6-\mathrm{H} 6$ & 0.9500 \\
\hline $\mathrm{C} 2-\mathrm{C} 3$ & $1.3965(13)$ & $\mathrm{C} 7-\mathrm{H} 7 \mathrm{~A}$ & 0.9800 \\
\hline $\mathrm{C} 2-\mathrm{H} 2$ & 0.9500 & $\mathrm{C} 7-\mathrm{H} 7 \mathrm{~B}$ & 0.9800 \\
\hline $\mathrm{C} 3-\mathrm{C} 2^{\mathrm{i}}$ & $1.3966(13)$ & $\mathrm{C} 7-\mathrm{H} 7 \mathrm{C}$ & 0.9800 \\
\hline $\mathrm{C} 3-\mathrm{H} 3$ & 0.9500 & $\mathrm{~B} 1-\mathrm{C}^{\mathrm{i}}$ & $1.4881(12)$ \\
\hline $\mathrm{C} 4-\mathrm{N} 1-\mathrm{C} 4$ & $120.78(11)$ & $\mathrm{C} 6-\mathrm{C} 5-\mathrm{C} 4$ & $119.88(9)$ \\
\hline $\mathrm{C} 4 \mathrm{i}-\mathrm{N} 1-\mathrm{B} 1$ & $119.61(6)$ & $\mathrm{C} 6-\mathrm{C} 5-\mathrm{H} 5$ & 120.1 \\
\hline $\mathrm{C} 4-\mathrm{N} 1-\mathrm{B} 1$ & $119.61(6)$ & $\mathrm{C} 4-\mathrm{C} 5-\mathrm{H} 5$ & 120.1 \\
\hline $\mathrm{C} 2-\mathrm{C} 1-\mathrm{B} 1$ & $117.56(9)$ & $\mathrm{C} 5^{\mathrm{i}}-\mathrm{C} 6-\mathrm{C} 5$ & $119.48(13)$ \\
\hline $\mathrm{C} 2-\mathrm{C} 1-\mathrm{H} 1$ & 121.2 & $\mathrm{C} 5^{\mathrm{i}}-\mathrm{C} 6-\mathrm{H} 6$ & 120.3 \\
\hline $\mathrm{B} 1-\mathrm{C} 1-\mathrm{H} 1$ & 121.2 & $\mathrm{C} 5-\mathrm{C} 6-\mathrm{H} 6$ & 120.3 \\
\hline $\mathrm{C} 1-\mathrm{C} 2-\mathrm{C} 3$ & $122.22(9)$ & $\mathrm{C} 4-\mathrm{C} 7-\mathrm{H} 7 \mathrm{~A}$ & 109.5 \\
\hline $\mathrm{C} 1-\mathrm{C} 2-\mathrm{H} 2$ & 118.9 & $\mathrm{C} 4-\mathrm{C} 7-\mathrm{H} 7 \mathrm{~B}$ & 109.5 \\
\hline $\mathrm{C} 3-\mathrm{C} 2-\mathrm{H} 2$ & 118.9 & $\mathrm{H} 7 \mathrm{~A}-\mathrm{C} 7-\mathrm{H} 7 \mathrm{~B}$ & 109.5 \\
\hline $\mathrm{C} 2-\mathrm{C} 3-\mathrm{C} 2^{\mathrm{i}}$ & $121.13(13)$ & $\mathrm{C} 4-\mathrm{C} 7-\mathrm{H} 7 \mathrm{C}$ & 109.5 \\
\hline $\mathrm{C} 2-\mathrm{C} 3-\mathrm{H} 3$ & 119.4 & $\mathrm{H} 7 \mathrm{~A}-\mathrm{C} 7-\mathrm{H} 7 \mathrm{C}$ & 109.5 \\
\hline $\mathrm{C} 22^{\mathrm{i}}-\mathrm{C} 3-\mathrm{H} 3$ & 119.4 & $\mathrm{H} 7 \mathrm{~B}-\mathrm{C} 7-\mathrm{H} 7 \mathrm{C}$ & 109.5 \\
\hline $\mathrm{N} 1-\mathrm{C} 4-\mathrm{C} 5$ & $119.99(9)$ & $\mathrm{C} 1-\mathrm{B} 1-\mathrm{C} 1^{\mathrm{i}}$ & $119.30(12)$ \\
\hline $\mathrm{N} 1-\mathrm{C} 4-\mathrm{C} 7$ & $118.55(9)$ & $\mathrm{C} 1-\mathrm{B} 1-\mathrm{N} 1$ & $120.35(6)$ \\
\hline $\mathrm{C} 5-\mathrm{C} 4-\mathrm{C} 7$ & $121.46(8)$ & $\mathrm{C} 1{ }^{\mathrm{i}}-\mathrm{B} 1-\mathrm{N} 1$ & $120.35(6)$ \\
\hline $\mathrm{B} 1-\mathrm{C} 1-\mathrm{C} 2-\mathrm{C} 3$ & $1.10(12)$ & $\mathrm{C} 4-\mathrm{C} 5-\mathrm{C} 6-\mathrm{C} 5^{\mathrm{i}}$ & $0.52(6)$ \\
\hline $\mathrm{C} 1-\mathrm{C} 2-\mathrm{C} 3-\mathrm{C} 2^{\mathrm{i}}$ & $-0.59(7)$ & $\mathrm{C} 2-\mathrm{C} 1-\mathrm{B} 1-\mathrm{C} 1^{\mathrm{i}}$ & $-0.53(6)$ \\
\hline $\mathrm{C} 4-\mathrm{N} 1-\mathrm{C} 4-\mathrm{C} 5$ & $0.52(6)$ & $\mathrm{C} 2-\mathrm{C} 1-\mathrm{B} 1-\mathrm{N} 1$ & $179.47(6)$ \\
\hline $\mathrm{B} 1-\mathrm{N} 1-\mathrm{C} 4-\mathrm{C} 5$ & $-179.47(6)$ & $\mathrm{C} 4-\mathrm{N} 1-\mathrm{B} 1-\mathrm{C} 1$ & $-98.80(6)$ \\
\hline $\mathrm{C} 4-\mathrm{N} 1-\mathrm{C} 4-\mathrm{C} 7$ & $-178.65(9)$ & $\mathrm{C} 4-\mathrm{N} 1-\mathrm{B} 1-\mathrm{C} 1$ & $81.20(6)$ \\
\hline $\mathrm{B} 1-\mathrm{N} 1-\mathrm{C} 4-\mathrm{C} 7$ & $1.35(9)$ & $\mathrm{C} 4-\mathrm{N} 1-\mathrm{B} 1-\mathrm{C} 1^{\mathrm{i}}$ & $81.20(6)$ \\
\hline $\mathrm{N} 1-\mathrm{C} 4-\mathrm{C} 5-\mathrm{C} 6$ & $-1.05(12)$ & $\mathrm{C} 4-\mathrm{N} 1-\mathrm{B} 1-\mathrm{C}^{\mathrm{i}}$ & $-98.80(6)$ \\
\hline $\mathrm{C} 7-\mathrm{C} 4-\mathrm{C} 5-\mathrm{C} 6$ & $178.10(7)$ & & \\
\hline
\end{tabular}

Symmetry code: (i) $-x+1, y,-z+3 / 2$. 